\title{
Anakinra for the Treatment of COVID-19-Associated Pericarditis: A Case Report
}

\author{
Hazan Karadeniz ${ }^{1}$ (D) Betül Ayça Yamak ${ }^{2}$ - Hasan Selçuk Özger ${ }^{3} \cdot$ Burak Sezenöz $^{2}$ - Abdurrahman Tufan ${ }^{1}$. \\ Giacomo Emmi ${ }^{4}$
}

Accepted: 21 July 2020 / Published online: 30 July 2020

(C) Springer Science+Business Media, LLC, part of Springer Nature 2020

\section{Dear Editor,}

Since its recognition in December 2019 as a cause of potentially severe pneumonia, SARS-CoV-2 infection has rapidly spread globally, causing a pandemic. Although it is an infectious disease, several distinct manifestations have been described in the setting of the new coronavirus disease (COVID)-19 resulting from the hyperimmune response caused by the virus [1]. The heart is frequently involved in COVID-19 and is associated with severe outcomes. Numerous distinct cardiac complications have been reported in COVID-19 patients, including arrhythmia, myocarditis, and coronary thrombosis [2]. Pericarditis is rarely reported in COVID-19 patients, and no reports are available on its treatment and outcome [3]. Herein, we describe the case of a patient affected by COVID-19 complicated by pericarditis successfully treated with the IL-1R antagonist anakinra.

A previously healthy 33-year-old male presented to the emergency department (ED) with progressive retrosternal chest pain for the previous 5 days. He described worsening of pain with sitting forward and nonresponse to diclofenac. He also reported severe low back pain that started 1 week before his arrival at the ED on April 16. The physical examination findings were as follows: pulse
90 beats per minute and regular, blood pressure 118/ $78 \mathrm{mmHg}$, oxygen saturation $97 \%$ whilst breathing ambient air, and temperature $37.9{ }^{\circ} \mathrm{C}$. The rest of the physical examination was unremarkable. The nasopharyngeal swab for SARS-CoV-2 tested positive. Blood tests revealed normal D-dimer $(0.26 \mathrm{ng} / \mathrm{mL}$, normal $<0.5)$ and highsensitivity troponin $\mathrm{T}(<5 \mathrm{ng} / \mathrm{L})$ and elevated $\mathrm{C}$-reactive protein (CRP, $73.8 \mathrm{mg} / \mathrm{dl}, n<5$ ), interleukin (IL)-6 levels $(43.6 \mathrm{pg} / \mathrm{mL}$, normal $<5)$, and lymphopenia $\left(1060 / \mathrm{mm}^{3}\right)$. Rheumatoid factor, antinuclear, and anti-extractable nuclear antigen antibodies tested negative. The patient was treated with oral hydroxychloroquine and moxifloxacin as per the local recommended COVID-19 protocol, along with analgesics. The hydroxychloroquine dose was $400 \mathrm{mg}$ bid the first day and then $200 \mathrm{mg}$ bid for 5 additional days. However, on the third day of hospitalization, chest pain did not improve and D-dimer increased to $3.15 \mathrm{mg} / \mathrm{mL}$. A 12-lead electrocardiogram (ECG) showed T-negative in D2, D3, and AVF derivations (more prominent in the inferior lateral derivations); biphasic $\mathrm{P}$ wave in $\mathrm{V} 1$ derivation and $\mathrm{J}$ wave in both $\mathrm{D} 3$ and V6 derivations; and incomplete right ventricular conduction delay in $\mathrm{V} 1$ derivation ( $\mathrm{rSr}$ pattern) (Fig. 1). The echocardiogram showed normal left ventricular function with
Hazan Karadeniz

drhazankaradeniz@hotmail.com

Betül Ayça Yamak

aycayamak@windowslive.com

Hasan Selçuk Özger

sozger@yahoo.com

Burak Sezenöz

drburaksezenoz@gmail.com

Abdurrahman Tufan

dratufan@hotmail.com
Giacomo Emmi

giacomo.emmi@unifi.it

1 Division of Rheumatology, Department of Internal Medicine, Gazi University Faculty of Medicine, 06100 Ankara, Turkey

2 Department of Cardiology, Gazi University Faculty of Medicine, Ankara, Turkey

3 Department of Infectious Disease, Gazi University Faculty of Medicine, Ankara, Turkey

4 Department of Experimental and Clinical Medicine, University of Florence, Florence, Italy 

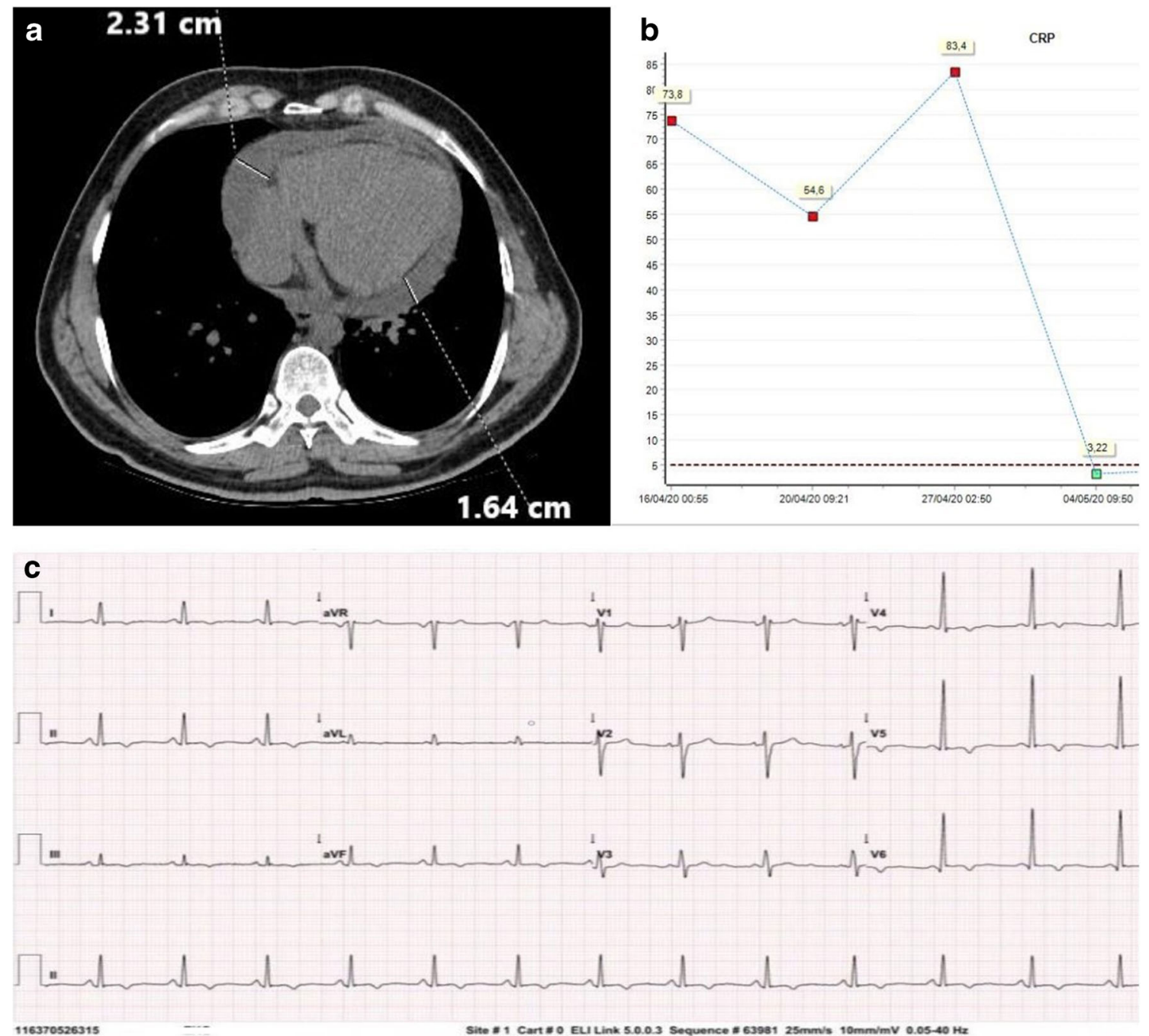

Fig. 1 (a) Thorax computed tomography showing pericardial effusion. (b) C-reactive protein time-table graph. (c) A 12-lead electrocardiogram (ECG) showing T-negative in D2, D3, and AVF derivations (more prominent in the inferior lateral derivations). Biphasic P wave in V1 derivation and $\mathrm{J}$ wave in both $\mathrm{D} 3$ and V6 derivations. Incomplete right ventricular conduction delay in $\mathrm{V} 1$ derivation (rSr pattern) circumferential pericardial effusion. Thorax computed tomography showed minimal ground-glass opacification, subpleural curvilinear lines, and pericardial effusion (greatest thickness $23.1 \mathrm{~mm}$ ), while it did not find indirect suggestive signs of pulmonary embolism. Nonetheless, enoxaparin $40 \mathrm{mg}$ twice daily was added to his treatment due to increased D-dimer. Given the clinical manifestations, laboratory results, and ECG findings, a diagnosis of pericarditis was made. A regimen of $0.5 \mathrm{mg}$ colchicine twice daily and $25 \mathrm{mg}$ indomethacin thrice daily was initiated on April 21. Five days later, fever and chest pain persisted, while CRP and D-dimer increased significantly to $83.4 \mathrm{mg} / \mathrm{L}$ and $5.65 \mathrm{ng} / \mathrm{mL}$, respectively, despite ongoing treatment with colchicine and indomethacin. Because his condition did not improve, subcutaneous administration of anakinra $100 \mathrm{mg} /$ day was started. Chest pain was promptly relieved. CRP and D-dimer values normalized 7 days after anakinra commencement, as well as echocardiogram. Anakinra was discontinued 7 days later and the patient was discharged in good clinical condition. He was doing well in his follow-up visit 2 weeks after the hospital discharge.

This is the first case in the literature showing the efficacy and safety of anakinra in a COVID-19-associated pericarditis after failure of colchicine therapy. Although rarely reported in COVID-19, pericarditis is an expected complication of viral infections. According to the 2015 European Society of Cardiology (ESC) Guidelines, diagnosis of pericarditis can be made using two of the following four criteria: (i) pericardial chest pain, (ii) widespread saddle-shaped or concave upward ST segment elevation or PR-segment depressions on ECG, (iii) new or worsening pericardial effusion, and (iv) pericardial 
friction rub that is auscultated by placing the diaphragm of the stethoscope over the left sternal border. Additional supportive findings were fever, positive inflammatory markers (leukocytosis, CRP), and evidence of pericardial inflammation by imaging [4]. Most patients with acute pericarditis have an idiopathic form, which accounts for more than $80 \%$ of cases [5]. According to recent findings, inflammasome activation is one of the main immunopathogenic pathways leading to pericardial inflammation. Interleukin (IL-1) $\beta$ is the predominant cytokine activated by inflammasomes and stimulates the synthesis of cyclooxygenase-2 (COX-2) and prostaglandins, thus leading to pericarditis [5]. Hence, treatments targeting inflammasome (colchicine), COX-2 (aspirin, ibuprofen, indomethacin), and IL-1 (anakinra) constitute the treatment options for idiopathic pericarditis [6]. Viral components and cytosolic danger signals such as mitochondrial injury, protein aggregates, and aberrant ion concentrations can activate NLR family pyrin domain containing 3 inflammasome, which in turn releases IL-1 $\beta$, IL-18, and the pro-pyroptotic factor gasdermin D [7]. In a previous study, SARS-CoV-2 was shown to activate the NLRP3 inflammasome and induce the production of IL-18 by human macrophages by its ion channel-forming E protein and ORF8b, which are also the structural components of SARS-CoV-2 [1]. Therefore, pericarditis is an expected clinical condition in COVID-19 [8]. COVID-19 is now considered a virus-induced immune disorder due to a constellation of features observed in cytokine storm syndromes. Hypercytokinemia is believed by many to be the main driver of morbidity and mortality in COVID-19 $[1,9]$. Therefore, anti-cytokine treatments such as tocilizumab (targeting IL-6) and anakinra (targeting IL-1) are being investigated for the treatment of patients with severe COVID-19 [1]. Based on these common pathogenetic mechanisms between idiopathic pericarditis and COVID-19, we suggest that the therapeutic approach in this clinical setting might be the same as for COVID-19-associated pericarditis [1]. In our case, we unsuccessfully tried colchicine and indomethacin, while obtaining rapid recovery with anakinra as for refractory cases of idiopathic recurrent pericarditis.

Nowadays, COVID-19-related cardiovascular disease in conjunction with lung involvement represents a leading cause of death and morbidity worldwide. Based on what we experienced in this case, anakinra could be an effective and reliable option in COVID-19-associated pericarditis owing to complete remission of pericarditis and prevention of long-term hospitalization, along with the absence of adverse effects.

Funding No funding was received for this paper.

\section{Compliance with Ethical Standards}

Conflict of Interest The authors declare that they have no conflict of interest.

Informed Consent Written informed consent was obtained from the patient.

\section{References}

1. Tufan A, Avanoğlu Güler A, Matucci-Cerinic M. COVID-19, immune system response, hyperinflammation and repurposing antirheumatic drugs. Turk J Med Sci. 2020;50(SI-1):620-32 Published 2020 Apr 21.

2. Kang Y, Chen T, Mui D, et al. Cardiovascular manifestations and treatment considerations in covid-19 [published online ahead of print, 2020 Apr 30]. Heart. 2020; heartjnl-2020-317056.

3. Cizgici AY, Zencirkiran Agus H, Yildiz M. COVID-19 myopericarditis: it should be kept in mind in today's conditions. Am J Emerg Med. 2020;S0735-6757(20):30310-7.

4. Adler Y, Charron P, Imazio, et al. ESC guidelines for the diagnosis and management of pericardial diseases: the force for the diagnosis and Management of Pericardial Diseases of the European Society of Cardiology (ESC) endorsed by: the European Association for Cardio-Thoracic Surgery (EACTS). Eur Heart J. 2015;42:2921-64.

5. Buckley LF, Viscusi MM, Van Tassell BW, Abbate A. Interleukin-1 blockade for the treatment of pericarditis. Eur Heart J Cardiovasc Pharmacother. 2018;4(1):46-53.

6. Brucato A, Emmi G, Cantarini L, et al. Management of idiopathic recurrent pericarditis in adults and in children: a role for IL-1 receptor antagonism. Intern Emerg Med. 2018;13(4):475-89.

7. Zhao C, Zhao W. NLRP3 Inflammasome-A Key Player in Antiviral Responses. Front Immunol. 2020; 11:211. Published 2020 Feb 18.

8. Hua A, O'Gallagher K, Sado D, Byrne J. Life-threatening cardiac tamponade complicating myo-pericarditis in COVID-19. Eur Heart J. 2020; ehaa253.

9. Aouba A, Baldolli A, Geffray L, et al. Targeting the inflammatory cascade with anakinra in moderate to severe COVID-19 pneumonia: case series [published online ahead of print, 2020 may 6]. Ann Rheum Dis. 2020;annrheumdis-2020-217706.

Publisher's Note Springer Nature remains neutral with regard to jurisdictional claims in published maps and institutional affiliations. 